\title{
Tactison: a Multimedia Learning Tool for Blind Children
}

\author{
Burger Dominique, Bouraoui Amina * \\ Mazurier Christian, Cesarano Serge, Sagot Jack ** \\ * INSERM-Creare - Université Pierre et Marie Curie, Paris, France. \\ E-mail : Dominique.Burger@snv.jussieu.fr \\ ** CNEFEI, Suresnes, France.
}

research supported by grant $n^{\circ}$ 89D0168 from the Ministère de la Recherche, and a research grant from the CNAMTS, Paris

\begin{abstract}
This paper introduces a tactile and auditory interactive material, Tactison, that was developed to teach blind or visually impaired children. The principles and design of the system are described. Some applications in education are presented and prospects for future developments are discussed. The purpose of this paper is to demonstrate how technology can bring about metaphorical environments which may be as useful for the blind as images are for sighted people.
\end{abstract}

\section{Introduction}

Images on paper play a central role in the education of sighted children, mainly because images provide useful substitutes for real objects. Pictures give children many opportunities to explore, identify, analyse, classify, and verbalise about things or scenes. Pencils, scissors, paste and other low cost tools allow them to transform and build up images.

In contrast, there are few educational materials available for visually handicapped children, and those thar are poorly-adapted, scarce and expensive. Blind children suffer from a lack of rich educational and recreational documents, even before they have learned to read and count.

This paper describes and discusses a computer interface designed to do two things : 1) enriching tactile learning support by embedding sounds, and 2) allowing the direct manipulation of data within a 2-D space. It was developed as part of a research project that was put in place by the Institut National de la Santé Et de la Recherche Médicale (INSERM) and the Centre National d'Etudes et de Formation pour l'Enfance Inadaptée (CNEFEI) to evaluate the potential of multimedia techniques in the education of blind children [Burger et al., 1990; Burger, 1991; Burger et al., 1991]. 


\section{Tactison}

\section{a) Metaphor}

Tactison was developed from the following metaphor used to devise : Imagine a tray containing objects which you cannot see because they are covered by a sheet of paper or cardboard, an overlay. You can, however, hear them since they emit a sound as soon as they are touched. They also produce different sounds, depending on the way you touch them, on whether you click, double-click or press. You can also move, cut and copy them. Overlays carry raised marks that can help locate, identify and retrieve objects and thus contribute to the understanding of the overall organisation. Some overlays are divided into areas, like windows on a screen. In fact these areas can be understood as outlining boxes on the tray in which the objects are or can be arranged. Boxes represent another type of object that can also utter messages when the finger clicks, double-clicks or presses them. But these boxes are stuck to the back of the tray. They therefore cannot be moved.

Obviously, this metaphor will never fully match the characteristics and functions of computer systems. Nevertheless, it seems to be fairly consistent with the everyday experiences of the blind, who perceive objects by hearing, reaching, manipulating and palpating. It may therefore help to create a representation of the system using intuitive knowledge and concepts.

\section{b) Hardware Implementation}

Tactison inherited an interaction technique previously explored at INSERM that combined a touch device and a sound production system, under the control of a desktop computer [Liard \& Burger, 1987]. A Concept Keyboard TM was used as touch device. This device has a $21 \times 29.7 \mathrm{~cm}$, A4-format and counts 256 active contacts whose lay-out is organised in 16-contact lines and 16-contact columns. Sounds are produced by either a PSOLA ${ }^{\text {TM }}$ or SYNTHE3 ${ }^{\text {TM }}$ French speaking text-to-speech synthesiser or a Sound Blaster ${ }^{\mathrm{TM}}$ sampling system. The minimum computer configuration is a $286 \mathrm{PC}$ with a $20 \mathrm{Mo}$ hard disk.

\section{c) Commands}

The basic operating concepts are: reading, page turning, marking, cutting, moving and copying. Each can apply to various objects, in various situations.

A read command makes it possible to get information about the contents of a line, the contents of a column, the contents of a box and all the contents on the tray

A turn page command allows users to change exercise by loading the contents of another tray

A mark command is used to make an object special or noticeable

A cut command removes an object from the tray

A move command changes the position of an object on the tray

A copy command creates a clone of an existing object in a specified position on the tray 


\section{d) Design Considerations}

\section{Tactile Overlays}

Some empirical rules of tactile design have been followed :

- very simple shapes for both abstract and figurative items,

- tactile hints and guidance

- symmetrical shapes as often as possible in order to make the pattern recognition process independent of the direction of finger exploration

Overlays are frequently revised after they have been used by the students

\section{Lay-out (Fig. 1)}

- Objects are always presented in the central of the overlay,

- Commands are presented at the periphery.

- If possible, things are presented in rows and columns in order to facilitate exploration. Once readers have understood this principle, they can guess the general organisation from only partial exploration. This can compensate for the loss of global perception. Such a regular arrangement also facilitates item retrieval.

\section{Actions}

Three types of physical actions can be carried out on the surface of an overlay: click, double-click and press. Each is precisely defined in time. A beep sound is emitted when a pressed action has been detected (time over $1.1 \mathrm{sec}$.).

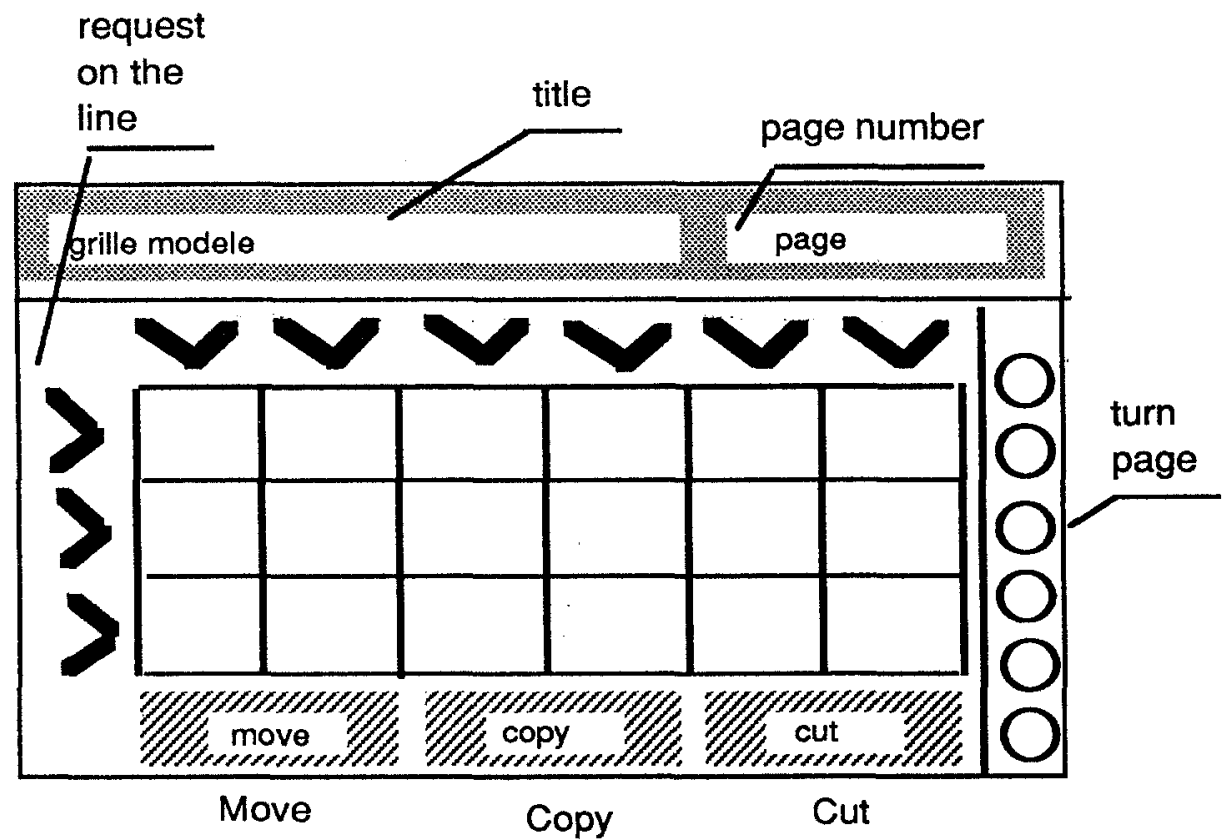

Fig. 1 The general lay-out adopted for the design of overlays. 


\section{Direct Manipulation}

All commands are accessible by means of keys on the overlay. Simple rules were adopted to allow an intuitive understanding of the commands and to facilitate their memorisation. For instance, the location of a read command corresponds to the objects to be read. As shown in figure 1, all buttons on the left part of the overlay are read-the-line-in-front-of-me commands, whereas buttons on the top are read-thecolumn-above-me commands. Clicking, double clicking or pressing the read command buttons address the different sounds that objects can emit. The cut, move and copy commands are activated by

1- pointing and clicking on an object,

2- pointing and clicking in the right location - if necessary,

3 - pointing and clicking on the command button.

The system responds by stating the action to be performed. The user can then confirm the command by double-clicking the command button.

\section{Sounds}

Both sampled sounds and synthesised spoken messages can be used. Their duration must be carefully chosen, depending on the type of activity, to allow clear identification or comprehension, but also to avoid being a load. We adopted the principle that if an action is to involve a sound utterance, any sound in progress is to be stopped.

\section{Semantic Organisation}

The messages are organized in a systematic semantic fashion, which means that the message associated with the click action can usually be described as giving a concrete, immediate aspect of evoked reality. The double-click sound generally provides more sophisticated information. The pressed sound can be a meta-message. For instance, let us imagine that the object to be evoked is a cat. The messages associated with its location could be:

click action: "miow" resulting from a sampling operation, double-click action: "My name is Pussy"

press action: "A cat is a domestic animal, it lives in a house with people".

\section{Discussion}

\section{a) Use in the Classroom}

An experiment was carried out for about a year wit carried out for about a year with both visually impaired and sighted children in two schools in the suburbs of Paris. It involved about 20 students ranging in age from 6 to 10 years (CP, CM2 in the French system). Several of the visually impaired children were totally blind. Students worked in small groups of 3 or 4 . Training sessions took place once a week and lasted about 40 minutes. Figure 2 shows an exercice in which students are asked to classify objects - a collection of animals - by moving them from one box to another. Two image boxes, Domestic Animals and Wild Animals have been dropped into the trash. The student must put them back into their original boxes. 


\section{b) The Students' Point of View}

The first point to come out of the experiment was the eagerness with which children participated in the weekly Tactison training sessions.

- Children learned to operate the device very rapidly. Children differentiated perfectly between the three pointing gestures on the touch device, clicking, double-clicking, pressing. (It is worth noting that gesture control does not necessarily imply the use of sophisticated or expensive devices.)

- The situations created with TactiSon entailed animated debates among students.

- The metaphor of mobile objects underneath the overlay was accepted without any difficulty.

- Students were able to work quite autonomously most of the time.

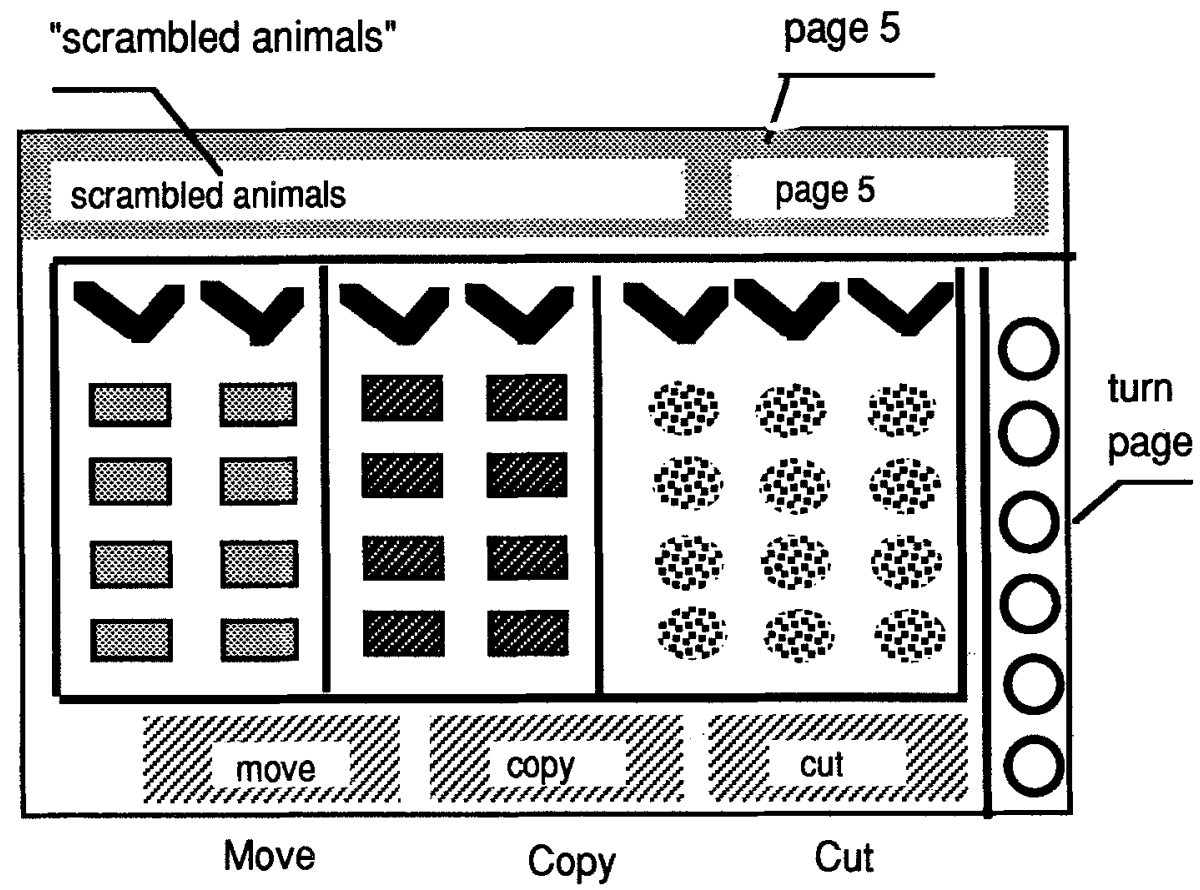

Fig. 2. The Scrambled Animals overlay illustrates how messages can give indications to the users. Message were uttered when an actions involved the corresponding objects.

\section{c) The Teachers' Point of View}

- Teachers appreciated the facilities dealing with different presentations of the same concept. It illustrated that transient auditory and tactile sensations can be used to contribute to the construction of more general and permanent representations of a concept. This is of great importance for the education of the blind, particularly since the overuse of verbal exchanges without connection to sensory experiences can lead to inadequate attitudes, known as verbalism, when facing real situations. 
- They also pointed out that this type of device offers rich opportunities to make children aware of spatial organisation and for training their ability to think about space. The use of a touch device proved to be an efficient way of retrieving and manipulating data, which confirmed an experimental study by Hill and Grieb [1988], in which subjects were given a stylus that could be used to access or manipulate auditory data organized on the surface of a pad device. They were asked to perform two tasks. One was to locate an area on a page, the other was a more classical editing task. The spatial device performed almost $50 \%$ better than a conventional keyboard-based access product.

- The device was considered to be "more practicable than manipulatives since it does not weigh $2 \mathrm{~kg}$ and since objects cannot be lost or thrown down".

- Many applications were suggested, including exercises and games which involve various types of material, such as letters, words, numbers, notes and melodies.

- Activities were principally organised in groups.

\section{d) The Designers Point of View}

- The design rules that emerged from this study were very specific and rarely obvious. Once a solution had been tested, it became a kind of standard for future interface design. Such rules also concerned tactile diagrams, sounds and interaction methods. Three concrete examples can be given:

a) the localisation and identification of tactile symbols often appears to be quite different from what visual experience suggests.

b) regular layout in rows and columns does not evoke such an orderly organisation when explored by hand as it does for vision.

c) as explained above, feedback messages are emitted before actions have been performed, while in visual interaction feedback is obtained by observing the moving image on the screen. This exemplifies how non-visual interaction sometimes has to be designed in a very special style.

- Despite the fact that speech production was obtained through a high-quality speech synthesiser, the variety of voice timbres appeared very poor, presenting some difficulties for some exercises. On the contrary, pre-sampled sounds were greatly appreciated by children.

\section{e) Future Prospects}

-We are now developing of an authoring tool that should satisfy the following need : the creation of tactile overlays, sound recording and editing, and the design of exercises, i.e. audio-tactiles and functions.

- New input/output peripherals should be added. For instance, a braille output would be easy and would enrich opportunities to develop reading and spelling skills.

- Automatic identification of the overlays, possibly by means of bar-coded marks or other techniques, could improve the interface by making the change of page automatic. 
- The addition of speech input and the implementation of multimodal interaction techniques would minimize the work load and avoid the frequent disruption of motor tasks involved in using a command buttons for the edition commands and the manipulation of data within the 2D-space [Salisbury, 1991; Martin \& Béroule, 1993, Bellik \& Burger, 1994].

\section{Conclusion}

This experiment leads to encouraging conclusions for the development of learning environments based on non-visual modalities, i.e. audition, touch and proprioception. It has confirmed that space provides a good support for the dynamic process of data organisation, comparison and classification. It is a way to help the user quickly find objects he/she needs. Semantic information, such as relative importance, similarity, or belongingness can also be communicated by the location of the objects in relation to each other.

Such multimedia environments appeared to be rich enough for teachers to consider them useful complements to existing techniques. It is hoped that they will help close the gap between the richness of learning materials available for the sighted and the relative scarcity of those accessible to the blind.

Finally, we feel that the interaction techniques that have been designed and tested in Tactison might also contribute to the design of non-visual interfaces for other computer applications.

\section{References}

1. Y. Bellik \& D. Burger : Multimodal interfaces: new solutions to the problem of computer accessibility for the blind. In : Proceedings of $\mathrm{CHI}^{\prime} 94$, Boston (1994)

2. D. Burger, E. Beltrando and J. Sagot : Sound Synthesis and Bar-code Technology to Develop Learning Environments for Blind Children. Journal of Visual Impairment and Blindness, 84, p.565-569 (1990)

3. D. Burger : Technologies hypermédias: Implications pour l'enseignement aux jeunes déficients visuels. Editions INSERM, Paris. 87p (1991)

4. D. Burger, J. Sagot et S. Cesarano : Technologie et outils d'enseignement spécialisés : Réflexions à partir du projet Polyson. Handicaps et inadaptations Les Cahiers du CTNERHI, 54, pp. 53-64 (1991)

5. D. R.Hill, and C. Grieb : Substitution for a Restricted Visual Channel in Multimodal Computer-Human Dialogue,IEEE Transactions on Systems Man and Cybernetics., vol. 18,2 , pp. 285-403 (1988) 
6. C. Liard et D. Burger : Composant audio-numérique et tactile et dispositif informatique en comportant application. Brevet INSERM $\mathrm{n}^{\circ} 8703340,11$ mars 1987 (1987)

7. J.C. Martin and D. Béroule : Trends in human-machine multi-modal interaction. In: Non-Visual Human-Computer Interactions, D. Burger and J.C.Sperandio, Eds., John-Libbey Eurotext, Montrouge, France, pp. 145-166 (1993)

8. M.W. Salisbury, J.H. Hendrickson, T.L. Mammers, C. Fu and S.A. Moody : Talk and Draw : Bundling Speech and Graphics, IEEE Computer, vol. 23, pp. 59-65 (1991) 